The Journal of Nonlinear $\mathbf{S}$ ciences and Applications

http://www.tjnsa.com

\title{
AN IMPLICIT METHOD FOR FUZZY PARABOLIC PARTIAL DIFFERENTIAL EQUATIONS
}

\author{
K. NEMATI ${ }^{1 *}$ AND M. MATINFAR ${ }^{2}$
}

\begin{abstract}
In this paper, we consider an implicit finite difference method for solving fuzzy partial differential equations (FPDEs). We present stability of this method and solve the parabolic equation with this scheme.
\end{abstract}

\section{INTRODUCTION}

Calculation of the solution of fuzzy partial differential equations is in general very difficult. We can find the exact solution only in some special cases. When we are studying in fields of physics and engineering, we often meet the problems of fuzzy partial differential equations where sometimes have to solving those as numerical methods. The topics of numerical methods for solving fuzzy differential equations have been rapidly growing in recent years. The concept of fuzzy numbers and arithmetic operations with these numbers were first introduced and investigated by Zadeh [11] and others. The fuzzy derivative was first introduced by S.L. Chang, Zadeh in [5]. The fuzzy differential equations and fuzzy initial value problem were regularly treated by O.Kaleva and S.Seikkala in [7, 8, 9]. J. Buckley and T. Feuring investigate exist of solution for FPDE in [4]. In [1, 2] T.Allahviranloo used a numerical method (explicit finite difference method) to solve FPDE, that was based on Seikkala derivative.

This paper is organized as follows:

In Section 2, we bring some basic definitions of fuzzy theory. In Section 3, we introduce fuzzy partial differential equations base on the Siekkala derivative. Finite difference method for fuzzy partial differential equation is discussed in Section 4.

Date: Received: 15 August 2008.

* Corresponding author.

2000 Mathematics Subject Classification. 65M06, 74S20, 94D05.

Key words and phrases. Parabolic boundary value problems, Fuzzy partial difference method, Implicit method. 
The necessary conditions will discuss for stability of the proposed method in Section 5. The implicit difference method is illustrated by solving an example in Section 6. We conclude in Section 7.

\section{Preliminaries}

- We placed a tilde $\operatorname{sign}(\sim)$ over a letter to denote a fuzzy number as subset of the real number.

- We write $\tilde{A}(x)$, a number in $[0,1]$, for the membership function of $\tilde{A}$ evaluated at $x$.

- An $\alpha$-cut of $\tilde{A}$, written $\tilde{A}[\alpha]$, is defined as $\{x \mid \tilde{A}(x) \geq \alpha\}$, for $0<\alpha \leq 1$.

- The triangular fuzzy number $\tilde{N}$ is defined by three number $a_{1}<a_{2}<a_{3}$, where the graph of $\tilde{N}(x)$, the membership function of the fuzzy number $\tilde{N}$, is a triangle with the base on the interval $\left[a_{1}, a_{3}\right]$ and vertex at $x=a_{2}$. We specify $\tilde{N}$ as $\left(a_{1}, a_{2}, a_{3}\right)$.

We write:

1. $\tilde{N}>0$ if $a_{1}>0$

2. $\tilde{N} \geq 0$ if $a_{1} \geq 0$;

3. $\tilde{N}<0$ if $a_{3}<0$;

4. $\tilde{N} \leq 0$ if $a_{3} \leq 0$.

We represent an arbitrary fuzzy number by an ordered pair of function $(\underline{u}(r), \bar{u}(r))$, $0 \leq r \leq 1$, which satisfies the following requirements:

1. $\underline{u}(r)$ is a bounded left continuous non decreasing function over $[0,1]$.

2. $\bar{u}(r)$ is a bounded right continuous non increasing function over $[0,1]$.

3. $\underline{u}(r) \leq \bar{u}(r), 0 \leq r \leq 1$. Where $\underline{u}(r)$ and $\bar{u}(r)$ are crisp number.

For arbitrary fuzzy number $x=(\underline{x}, \bar{x}), y=(\underline{y}, \bar{y})$ and real number $k$ :

1. $x=y$ if and only if $\underline{x}(r)=\underline{y}(r)$, and $\bar{x}(r)=\bar{y}(r)$ for all $0 \leq r \leq 1$.

2. $k x= \begin{cases}(k \underline{x}, k \bar{x}) & k \geq 0, \\ (k \bar{x}, k \underline{x}) & k<0 .\end{cases}$

3. $x+y=(\underline{x}(r)+\underline{y}(r), \bar{x}(r)+\bar{y}(r))$.

4. $x-y=(\underline{x}(r)-\overline{\bar{y}}(r), \bar{x}(r)-y(r))$.

5. $x \cdot y=\{\min \{\underline{x}(r) \cdot \underline{y}(r), \underline{x}(r) \cdot \bar{y}(r), \bar{x}(r) \cdot \underline{y}(r), \bar{x}(r) \cdot \bar{y}(r)\}, \max \{\underline{x}(r) \cdot \underline{y}(r), \underline{x}(r) \cdot \bar{y}(r), \bar{x}(r) \cdot \underline{y}(r), \bar{x}(r) \cdot \bar{y}$

Since the $\alpha$-cut of fuzzy numbers are always a closed and bounded, intervals, so we can write $\tilde{N}[\alpha]=[\underline{N}(\alpha), \bar{N}(\alpha)]$, for all $\alpha$.

Now we denote the set of all nonempty compact subset of $R^{n}$ by $\kappa^{n}$ and the subset of $\kappa^{n}$ consisting of nonempty convex compact sets by $\kappa_{c}^{n}$. Recall that

$$
\rho(x, A)=\min _{a \in A}\|x-a\|,
$$

is the distance of a point $x \in R^{n}$ from $A \in \kappa^{n}$ and also the Hausdorff separation $\rho(A, B)$ of $A, B \in \kappa^{n}$ is defined as

$$
\rho(A, B)=\max _{a \in A} \rho(a, B) .
$$


Note that the notation is consistent, since $\rho(a, B)=\rho(\{a\}, B)$. Now, $\rho$ is not a metric. In fact, $\rho(A, B)=0$ if and only is $A \subseteq B$.

The Hausdorff metric $d_{H}$ on $\kappa^{n}$ is defined by

$$
d_{H}(A, B)=\max \{\rho(A, B), \rho(B, A)\},
$$

and $\left(\kappa^{n}, d_{H}\right)$ is a complete metric space.

Let $E$ be the set of all upper semicontinuous normal convex fuzzy numbers with bounded $\alpha$-level sets. The metric $d_{H}$ is defined on $E$ as

$$
d_{\infty}(\tilde{u}, \tilde{v})=\sup \left\{d_{H}(\tilde{u}[\alpha], \tilde{v}[\alpha]): 0 \leq r \leq 1\right\}, \tilde{u}, \tilde{v} \in E .
$$

\section{Finite DiffEREnCE METHOD}

Assume $\tilde{U}$ is a fuzzy function of the independent crisp variables $x$ and $t$. Subdivided the $x-t$ plane into sets of equal rectangles of sides $\delta x=h, \delta t=k$, by equally space grid lines parallel to $O y$, defined by $x_{i}=i h, i=0,1,2, \ldots$ and equally spaced grid lines parallel to $O x$, defined by $y_{j}=j=0,1,2, \ldots$. Denote the value of $\tilde{U}$ at the representative mesh point $p(i h, j k)$ by

$$
\tilde{U}_{p}=\tilde{U}(i h, j k)=\tilde{U}_{i, j}
$$

and also denote the parametric form of fuzzy number, $\tilde{U}_{i, j}$ as follow

$$
\tilde{U}_{i, j}=\left(\underline{U}_{i, j}, \bar{U}_{i, j}\right) .
$$

Then by taylor's theorem and definition of standard difference $\left(D_{x} D_{x}\right) \tilde{U}_{i, j}=$ $\left(\underline{\left(D_{x} D_{x}\right) \tilde{U}_{i, j}}, \overline{\left(D_{x} D_{x}\right) \tilde{U}_{i, j}}\right)$,

where

$$
\begin{aligned}
& \frac{\left(D_{x} D_{x}\right) \tilde{U}_{i, j}}{} \simeq \frac{\underline{u}\{(i+1) h, j k\}-2 \bar{u}\{i h, j k\}+\underline{u}\{(i-1) h, j k\}}{h^{2}}, \\
& \overline{\left(D_{x} D_{x}\right) \tilde{U}_{i, j}} \simeq \frac{\bar{u}\{(i+1) h, j k\}-2 \underline{u}\{i h, j k\}+\bar{u}\{(i-1) h, j k\}}{h^{2}} .
\end{aligned}
$$

By (10) we have

$$
\begin{aligned}
& \frac{\left(D_{x} D_{x}\right) \tilde{U}_{i, j}}{2} \simeq \frac{\underline{u}_{i+1, j}-2 \bar{u}_{i, j}+\underline{u}_{i-1, j}}{h^{2}}, \\
& \overline{\left(D_{x} D_{x}\right) \tilde{U}_{i, j}} \simeq \frac{\bar{u}_{i+1, j}-2 \underline{u}_{i, j}+\bar{u}_{i-1, j}}{h^{2}} .
\end{aligned}
$$

with a leading error of order $h^{2}$. Similarly, with this notation the forwarddifference approximation for $\left(D_{t} \tilde{U}\right)$ at $p$ is

$$
\begin{aligned}
& \frac{\left(D_{t}\right) \tilde{U}_{i, j}}{} \simeq \frac{\underline{u}_{i, j+1}-\bar{u}_{i, j}}{k}, \\
& \overline{\left(D_{t}\right) \tilde{U}_{i, j}} \simeq \frac{\bar{u}_{i, j+1}-\underline{u}_{i, j}}{k},
\end{aligned}
$$


with a leading error of $O(k)$. By (11) and (12) and technique of Crank-Nicolson $(C-N)$, the finite difference representation of equation

$$
\left(D_{t}\right) \tilde{U}=\beta^{2}\left(D_{x} D_{x}\right) \tilde{U}
$$

is

$$
\frac{\tilde{U}_{i, j+1}-\tilde{U}_{i, j}}{k}=\frac{\beta^{2}}{2}\left\{\frac{\tilde{U}_{i+1, j+1}-2 \tilde{U}_{i, j+1}+\tilde{U}_{i-1, j+1}}{h^{2}}+\frac{\tilde{U}_{i+1, j}-2 \tilde{U}_{i, j}+\tilde{U}_{i-1, j}}{h^{2}}\right\},
$$

or the following equations must be hold:

$$
\left\{\begin{array}{l}
\underline{u}_{i, j+1}-\lambda \underline{u}_{i-1, j+1}-\lambda \bar{u}_{i+1, j+1}+2 \lambda \bar{u}_{i, j+1}=(1-2 \lambda) \bar{u}_{i, j}+\lambda \underline{u}_{i-1, j}+\lambda \underline{u}_{i+1, j} \\
\bar{u}_{i, j+1}-\lambda \bar{u}_{i-1, j+1}-\lambda \underline{u}_{i+1, j+1}+2 \lambda \underline{u}_{i, j+1}=(1-2 \lambda) \underline{u}_{i, j}+\lambda \bar{u}_{i-1, j}+\lambda \bar{u}_{i+1, j}
\end{array}\right.
$$

where $\tilde{U}=(\underline{u}, \bar{u})$ is the exact solution of the approximating difference equations, $x_{i}=i h,(i=0,1,2, \ldots, n)$ and $t_{j}=j k,(j=0,1,2, \ldots)$, and $\lambda=\frac{\beta^{2} k}{2 h^{2}}$.

\section{Finite difference Method}

Assume $\tilde{U}$ is a fuzzy function of the independent crisp variables $x$ and $t$. Subdivided the $x-t$ plane into sets of equal rectangles of sides $\delta x=h, \delta t=k$, by equally space grid lines parallel to $O y$, defined by $x_{i}=i h, i=0,1,2, \ldots$ and equally spaced grid lines parallel to $O x$, defined by $y_{j}=j=0,1,2, \ldots$. Denote the value of $\tilde{U}$ at the representative mesh point $p(i h, j k)$ by

$$
\tilde{U}_{p}=\tilde{U}(i h, j k)=\tilde{U}_{i, j}
$$

and also denote the parametric form of fuzzy number, $\tilde{U}_{i, j}$ as follow

$$
\tilde{U}_{i, j}=\left(\underline{U}_{i, j}, \bar{U}_{i, j}\right) .
$$

Then by taylor's theorem and definition of standard difference $\left(D_{x} D_{x}\right) \tilde{U}_{i, j}=$ $\left(\left(D_{x} D_{x}\right) \tilde{U}_{i, j}, \overline{\left(D_{x} D_{x}\right) \tilde{U}_{i, j}}\right)$,

where

$$
\begin{aligned}
& \frac{\left(D_{x} D_{x}\right) \tilde{U}_{i, j}}{} \simeq \frac{\underline{u}\{(i+1) h, j k\}-2 \bar{u}\{i h, j k\}+\underline{u}\{(i-1) h, j k\}}{h^{2}}, \\
& \overline{\left(D_{x} D_{x}\right) \tilde{U}_{i, j}} \simeq \frac{\bar{u}\{(i+1) h, j k\}-2 \underline{u}\{i h, j k\}+\bar{u}\{(i-1) h, j k\}}{h^{2}} .
\end{aligned}
$$

By (10) we have

$$
\begin{aligned}
& \frac{\left(D_{x} D_{x}\right) \tilde{U}_{i, j}}{} \simeq \frac{\underline{u}_{i+1, j}-2 \bar{u}_{i, j}+\underline{u}_{i-1, j}}{h^{2}}, \\
& \overline{\left(D_{x} D_{x}\right) \tilde{U}_{i, j}} \simeq \frac{\bar{u}_{i+1, j}-2 \underline{u}_{i, j}+\bar{u}_{i-1, j}}{h^{2}} .
\end{aligned}
$$


with a leading error of order $h^{2}$. Similarly, with this notation the forwarddifference approximation for $\left(D_{t} \tilde{U}\right)$ at $p$ is

$$
\begin{aligned}
& \frac{\left(D_{t}\right) \tilde{U}_{i, j}}{} \simeq \frac{\underline{u}_{i, j+1}-\bar{u}_{i, j}}{k}, \\
& \overline{\left(D_{t}\right) \tilde{U}_{i, j}} \simeq \frac{\bar{u}_{i, j+1}-\underline{u}_{i, j}}{k},
\end{aligned}
$$

with a leading error of $O(k)$. By (11) and (12) and technique of Crank-Nicolson $(C-N)$, the finite difference representation of equation

$$
\left(D_{t}\right) \tilde{U}=\beta^{2}\left(D_{x} D_{x}\right) \tilde{U}
$$

is

$$
\frac{\tilde{U}_{i, j+1}-\tilde{U}_{i, j}}{k}=\frac{\beta^{2}}{2}\left\{\frac{\tilde{U}_{i+1, j+1}-2 \tilde{U}_{i, j+1}+\tilde{U}_{i-1, j+1}}{h^{2}}+\frac{\tilde{U}_{i+1, j}-2 \tilde{U}_{i, j}+\tilde{U}_{i-1, j}}{h^{2}}\right\},
$$

or the following equations must be hold:

$$
\left\{\begin{array}{l}
\underline{u}_{i, j+1}-\lambda \underline{u}_{i-1, j+1}-\lambda \bar{u}_{i+1, j+1}+2 \lambda \bar{u}_{i, j+1}=(1-2 \lambda) \bar{u}_{i, j}+\lambda \underline{u}_{i-1, j}+\lambda \underline{u}_{i+1, j} \\
\bar{u}_{i, j+1}-\lambda \bar{u}_{i-1, j+1}-\lambda \underline{u}_{i+1, j+1}+2 \lambda \underline{u}_{i, j+1}=(1-2 \lambda) \underline{u}_{i, j}+\lambda \bar{u}_{i-1, j}+\lambda \bar{u}_{i+1, j}
\end{array}\right.
$$

where $\tilde{U}=(\underline{u}, \bar{u})$ is the exact solution of the approximating difference equations, $x_{i}=i h,(i=0,1,2, \ldots, n)$ and $t_{j}=j k,(j=0,1,2, \ldots)$, and $\lambda=\frac{\beta^{2} k}{2 h^{2}}$.

\section{A Necessary Condition for Stability}

Now we are going to consider the stability of the classical implicit equations (14). If the boundary values are know at $i=0$ and $N, j>0$, then the $2(N-1)$ equations can be written in matrix as

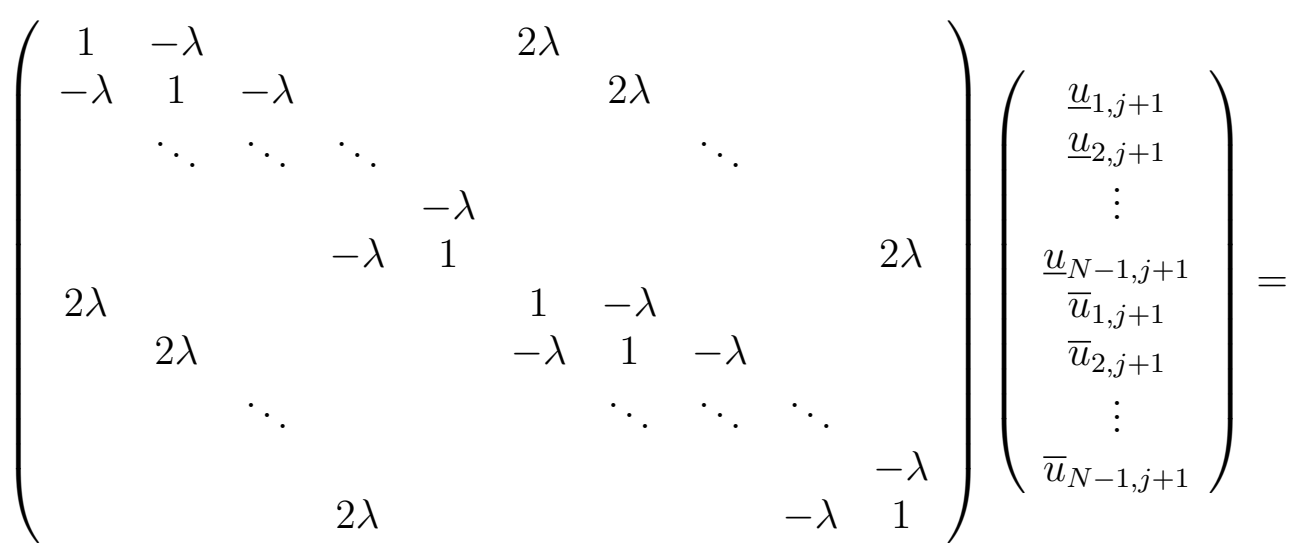




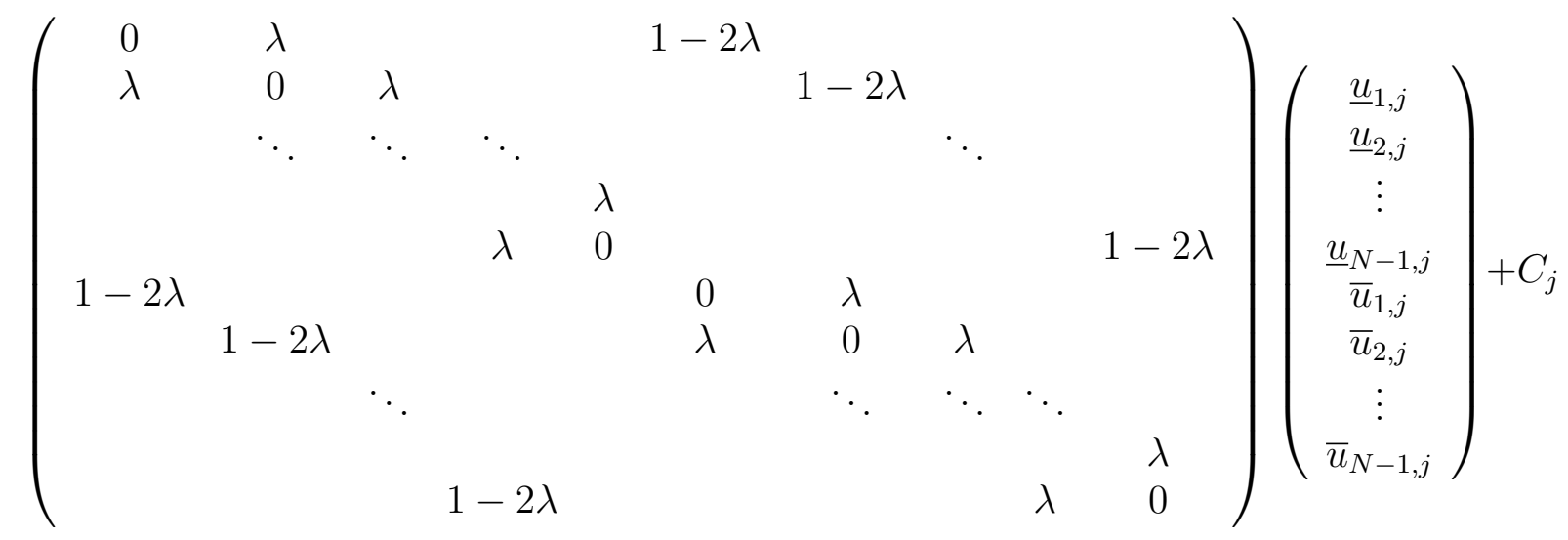

i.e,

$$
P\left(\begin{array}{c}
\underline{w}_{j+1} \\
\bar{w}_{j+1}
\end{array}\right)=Q\left(\begin{array}{c}
\underline{w}_{j} \\
\bar{w}_{j}
\end{array}\right)+C_{j}
$$

where

$P=\left(\begin{array}{cc}A & B \\ B & A\end{array}\right), A=I-\lambda\left(\begin{array}{ccccc}0 & 1 & & & \\ 1 & 0 & 1 & & \\ & \ddots & \ddots & \ddots & \\ & & 1 & 0 & 1 \\ & & & 1 & 0\end{array}\right), B=2 \lambda\left(\begin{array}{cccc}1 & & & \\ & 1 & & \\ & & \ddots & \\ & & & 1\end{array}\right)=2 \lambda I$

and

$Q=\left(\begin{array}{cc}E & F \\ F & E\end{array}\right), F=\lambda\left(\begin{array}{ccccc}0 & 1 & & & \\ 1 & 0 & 1 & & \\ & \ddots & \ddots & \ddots & \\ & & 1 & 0 & 1 \\ & & & 1 & 0\end{array}\right), E=(1-2 \lambda)\left(\begin{array}{cccc}1 & & & \\ & 1 & & \\ & & \ddots & \\ & & & 1\end{array}\right)=(1-2 \lambda) I$ Thus

$$
\left(\begin{array}{c}
\underline{w}_{j+1} \\
\bar{w}_{j+1}
\end{array}\right)=P^{-1} Q\left(\underline{\underline{w}}_{j}\right)+P^{-1} C_{j}, T=P^{-1} Q
$$

We first give Theorem 4.1 and then prove the stability of the above method in Theorem 4.2 . 
Theorem 4.1 Let matrix $P$ has spacial structure as follow

$$
\left(\begin{array}{cc}
A & B \\
B & A
\end{array}\right)
$$

Then the eigenvalues of $P$ are union of eigenvalues of $A+B$ and eigenvalues of $A-B[3$.

Now we prove the stability of $C-N$ method in the following theorem.

Theorem 4.2 Difference equations (14) for all $\lambda$ are stable.

Proof: It is sufficient to show that $\rho(T)<1$ in (15). Thus, by Theorem 4.1, it is enough to find eigenvalues of $A+B$ and $A-B$, where

$$
A+B=\left(\begin{array}{ccccc}
1+2 \lambda & -\lambda & & & \\
-\lambda & 1+2 \lambda & -\lambda & & \\
& \ddots & \ddots & \ddots & \\
& & & & -\lambda \\
& & & -\lambda & 1+2 \lambda
\end{array}\right)
$$

and

$$
A-B=\left(\begin{array}{ccccc}
1-2 \lambda & -\lambda & & & \\
-\lambda & 1-2 \lambda & -\lambda & & \\
& \ddots & \ddots & \ddots & \\
& & & & -\lambda \\
& & & -\lambda & 1-2 \lambda
\end{array}\right)
$$

Let matrixes $(N-1) \times(N-1) S$ and $S^{\prime}$ as follow

$$
S=\left(\begin{array}{ccccc}
2 & -1 & & & \\
-1 & 2 & -1 & & \\
& \ddots & \ddots & \ddots & \\
& & & & -1 \\
& & & -1 & 2
\end{array}\right), S^{\prime}=\left(\begin{array}{ccccc}
2 & 1 & & & \\
1 & 2 & 1 & & \\
& \ddots & \ddots & \ddots & \\
& & & & 1 \\
& & & 1 & 2
\end{array}\right)
$$

Thus

$$
\begin{aligned}
& A+B=I+\lambda S, \\
& A-B=I-\lambda S^{\prime} .
\end{aligned}
$$

where $I$ is the Unit matrix of order $(N-1)$ and $S, S^{\prime}$ are $(N-1) \times(N-1)$ matrixes whose eigenvalues $\lambda_{S}$ and $\lambda_{S^{\prime}}$ are given by [10]

$$
\begin{aligned}
\lambda_{S}= & \lambda_{S^{\prime}}=2+2 \cos \frac{k \pi}{N}=2\left(1+\cos \frac{k \pi}{N}\right) \\
& =4 \cos ^{2} \frac{k \pi}{N}, k=1,2, \ldots, N-1 .
\end{aligned}
$$


Hence, the eigenvalues of $A+B$ and $A-B$ are

$$
\begin{aligned}
& \lambda_{A+B}=1+4 \lambda \cos ^{2} \frac{k \pi}{2 N}, \\
& \lambda_{A-B}=1+4 \lambda \cos ^{2} \frac{k \pi}{2 N},
\end{aligned}
$$

where at those $k=1,2, \ldots, N-1$, respectively

$$
E+F=\left(\begin{array}{ccccc}
1-2 \lambda & \lambda & & & \\
\lambda & 1-2 \lambda & \lambda & & \\
& \ddots & \ddots & \ddots & \\
& & & \lambda & \lambda \\
& & & \lambda & 1-2 \lambda
\end{array}\right)
$$

and

$$
E-F=\left(\begin{array}{ccccc}
2 \lambda-1 & \lambda & & & \\
\lambda & 2 \lambda-1 & \lambda & & \\
& \ddots & \ddots & \ddots & \lambda \\
& & & & \lambda \\
& & & \lambda-1
\end{array}\right)
$$

thus

$$
\begin{gathered}
E+F=I-\lambda S, \\
E-F=-I+\lambda S^{\prime} .
\end{gathered}
$$

The eigenvalues for above matrixes are:

$$
\begin{gathered}
\lambda_{E+F}=1-4 \lambda \cos ^{2} \frac{k \pi}{2 N}, k=1,2, \ldots, N-1 \\
\lambda_{E-F}=-1+4 \lambda \cos ^{2} \frac{k \pi}{2 N}, k=1,2, \ldots, N-1
\end{gathered}
$$

We know when $\rho(T)=\rho\left(P^{-1} Q\right)<1$, then iterative method is stable.

$$
\rho(T)=\max _{k}\left|\frac{1-4 \lambda \cos ^{2} \frac{k \pi}{2 N}}{1+4 \lambda \cos ^{2} \frac{k \pi}{2 N}}\right|<1,
$$

or

$$
\rho(T)=\max _{k}\left|\frac{-1+4 \lambda \cos ^{2} \frac{k \pi}{2 N}}{1+4 \lambda \cos ^{2} \frac{k \pi}{2 N}}\right|<1,
$$

for all $\lambda$. Therefore, the scheme is stable. 


\section{EXAMPLE}

Consider the fuzzy parabolic partial differential equation

$$
\frac{\partial \tilde{U}}{\partial t}(x, t)=\frac{\partial^{2} \tilde{U}}{\partial x^{2}}(x, t), 0 \leq x \leq 1, t>0,
$$

with the boundary conditions

$$
\tilde{U}(0, t)=\tilde{U}(1, t)=0, t>0
$$

and

$$
\tilde{U}(x, 0)=\tilde{f}(x)=\tilde{K} \sin (\pi x), 0 \leq x \leq 1,
$$

and

$$
\tilde{K}=[\underline{k}(\alpha), \bar{k}(\alpha)]=[\alpha-1,1-\alpha] .
$$

The exact solution for

$$
\begin{aligned}
& \frac{\partial \underline{U}}{\partial t}(x, t ; \alpha)=\frac{\partial^{2} \underline{U}}{\partial x^{2}}(x, t ; \alpha), \\
& \frac{\partial \bar{U}}{\partial t}(x, t ; \alpha)=\frac{\partial^{2} \bar{U}}{\partial x^{2}}(x, t ; \alpha),
\end{aligned}
$$

for $0 \leq x \leq 1, t>0$ are $\underline{U}(x, y ; \alpha)=\underline{k}(\alpha) e^{-\pi^{2} t} \sin (\pi x)$ and $\bar{U}(x, y ; \alpha)=$ $\bar{k}(\alpha) e^{-\pi^{2} t} \sin (\pi x)$.

We use the equations (14) to approximate the exact solution. We have shown in Figure1 and Figure2 some of the $\tilde{U}$ value in the various grid points for all $\alpha$ belong to $[0,1]$. In these Figures have compared approximated solutions and the exact solutions, where Hausdorff distance between the solutions in the case $h=0.01, k=0.001$, and $\lambda=5$, is 0.00115909722312 .

Symbols in the Figures are:

$\tilde{U}_{1,1}:\left\{\begin{array}{l}\ldots: \text { approx } \\ \square \text { : exact, }\end{array} \quad \tilde{U}_{5,1}:\left\{\begin{array}{l}-.- \text { : approx } \\ + \text { : exact, }\end{array} \quad \tilde{U}_{2,2}:\left\{\begin{array}{l}--: \text { approx } \\ \triangle \text { : exact },\end{array} \quad \tilde{U}_{3,4}:\left\{\begin{array}{l}-: \text { approx } \\ 0: \text { exact },\end{array}\right.\right.\right.\right.$

\section{CONClusion}

The fuzzy partial differential equation can be applied for modeling in physics, engineering and mechanical systems. In this paper, we applied an implicit finite difference method to solve some partial differential equations. Moreover, the numerical examples gave a good approximation for the solutions. 


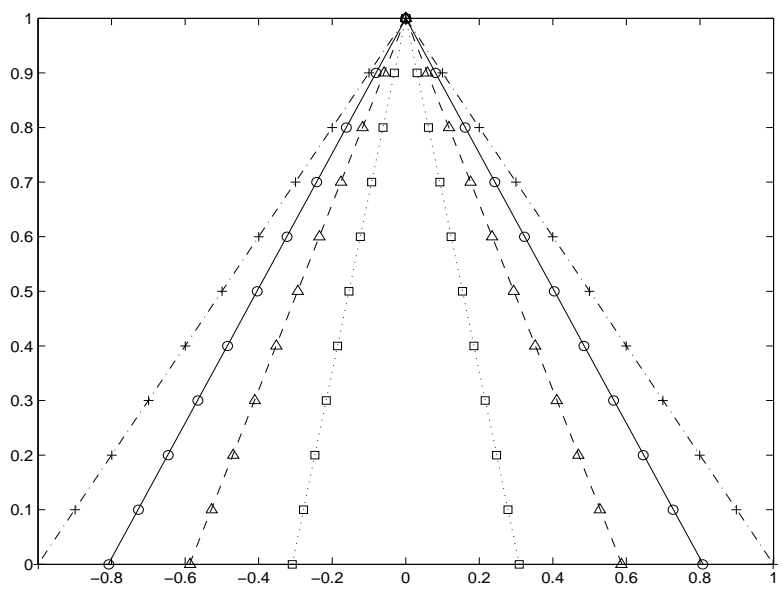

Figure $1 . h=0.1, k=0.0001$ and $\lambda=0.005$.

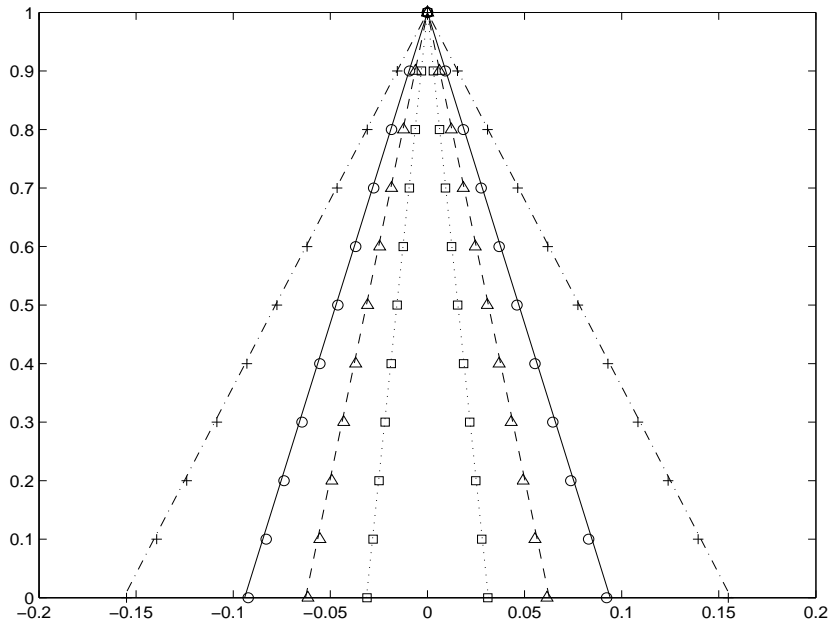

Figure $2 . h=0.01, k=0.001$ and $\lambda=5$

\section{REFERENCES}

[1] T. Allahviranloo, Difference methods for fuzzy partial differential equations, Computational Methods in Applied Mathematics, 2 (3) (2002) 233-242. 1

[2] T. Allahviranloo, M. Afshar, Difference method for solving the fuzzy parabolic equations, Applied Mathematical Sciences, 1 (27) (2007) 1299-1309. 1

[3] T. Allahviranloo, N. Ahmadi, E. Ahmadi, Kh. Shams Alketabi, Block Jacobi two-stage method for fuzzy systems of linear equations, Applied Mathematics and Computation 175 (2006) 1217-1228. 5 
[4] J.J. Buckley, T. Feuring, Introduction to fuzzy partial differential equations, Fuzzy Sets and Systems 105 (1999) 241-248. 1

[5] S.L. Chang, L.A. Zadeh, On fuzzy mapping and control, IEEE Thrans, System Man Cybernet. 2 (1972) 30-34. 1

[6] D. Dubois, H. Prade, Towards fuzzy differential calculus: Part3, differentiation Fuzzy Sets and Systems 8 (1982) 225-233.

[7] O. Kaleva, Fuzzy differential equations, Fuzzy Sets and Systems 24 (1987) 301-317. 1

[8] O. Kaleva, The cuachy problem for fuzzy differential equations, Fuzzy Sets and Systems 35 (1990) 389-396. 1

[9] S. Seikkala, on the fuzzy initial value problem, Fuzzy Sets and Systems 24 (1987) 319-330. 1

[10] G.D. Smith, Numerical Solution of Partial Differential Equations, 1993. 5

[11] L.A. Zadeh, The concept of a linguistic variable and its application to approximate reasoning, Information Science 8 (1975) 199-249. 1

1 Islamic Azad University, Nur Branch, Nur, Iran.

E-mail address: knms81@gmail.com

1 Department of Mathematics, University of Mazandaran, Babolsar, Iran.

E-mail address: m.matinfar@umz.ac.ir 